\title{
On Dynamic Wavelength Assignment in WDM Optical Networks
}

\author{
Murat Alanyali
}

Department of Electrical and Electronics Engineering

Bilkent University, Ankara, 06533 Turkey

E-mail: alanyali@ee.bilkent.edu.tr

\section{Contents}

1 Introduction $\quad 1$

2 Loss Networks $\quad 4$

2.1 Networks without wavelength continuity constraint . . . . . . . 5

2.2 Networks with wavelength continuity constraint . . . . . . . . 6

3 Nonblocking Networks $\quad 13$

4 Conclusion $\quad 14$

References

\section{Introduction}

Optical fiber has been used as the physical medium for high rate data transmission since late 1960s. Early applications of optical fiber communications modulated data onto a single optical carrier frequency that is commonly referred to as a wavelength. The carried data rate is therefore limited by the speed of electronics that generate the signal, thereby grossly underutilizing the tens of $\mathrm{THz}$ of useful bandwidth available on the fiber[1]. The Wavelength Division Multiplexing (WDM) technology now allows multiplexing 


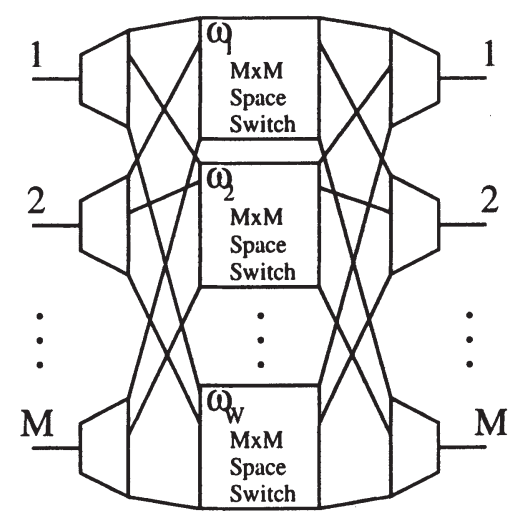

(a)

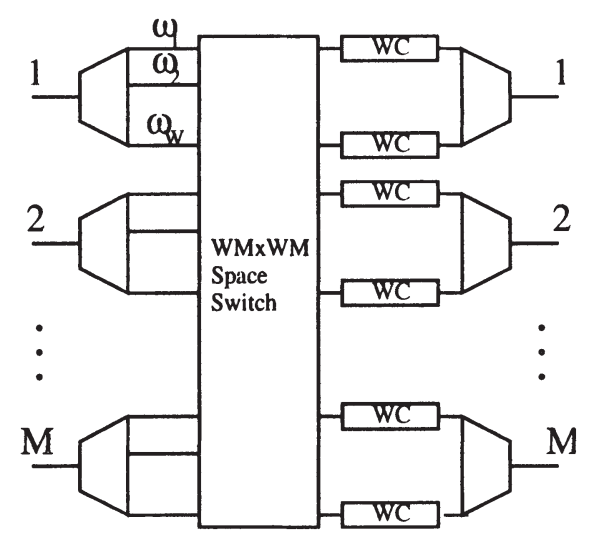

(b)

Figure 1: Structure of (a) a wavelength selective (WS) optical switch, and (b) a wavelength converter (WC) optical switch.

several optical carriers on the same fiber, opening up the available potential. Current deployments of WDM are based on point-to-point links. This entails demodulating the optical signal at each switching node and carrying out switching electronically. Remarkable increase in the carried data rates due to the WDM technology make these switches inherent bottlenecks in the network. One promising approach to solve this problem is optical switching which entails switching entire wavelengths without any electronic processing.

Optical switches have varying capabilities with respect to wavelength conversion. At one extreme, a wavelength selective (WS) switch offers no conversion capability: the signal on a particular wavelength cannot be modulated onto a different wavelength as it passes through the switch. Hence in a network employing exclusively WS switches, an end-to-end connection is established by using the same wavelength on each link of its path. This condition is referred to as the wavelength continuity constraint. Figure 1.a gives the structure of a WS switch with $M$ input and $M$ output fibers. Throughout the paper it is assumed that the same set of wavelengths is available on all fibers. At the other extreme, a wavelength converter $(W C)$ switch can remodulate the data on any inbound wavelength onto any outbound wavelength. WC switches remove the wavelength continuity constraint and therefore result in better utilization of the fiber capacity. For example in the network of Figure 2 with two wavelengths $w_{1}, w_{2}$ on each fiber, if fiber 


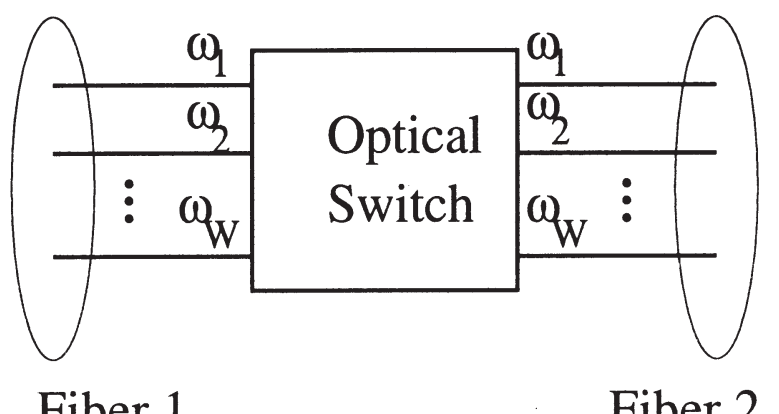

Figure 2: A simple topology with one optical switch and two fibers.

1 carries one connection which fully utilizes $w_{1}$ and fiber 2 carries another connection which fully utilizes $w_{2}$, a third connection that uses both fibers can be established only if the optical switch is a WC. The architecture of a WC switch is given in Figure 1.b. The wavelength converters in the figure ideally have the capability of providing conversion between any pair of wavelengths. However currently obtained conversion performance strongly depends on the specific wavelength pair, and technology is yet to mature to allow full conversion capability[2]. This practical consideration gives rise to the limited wavelength converter switch, which stands somewhere between WS and WC models, and provides conversion only between specified wavelength pairs. The architecture of the switch is the same as that of a WC switch except for the reduced capability of the converters.

A main theme of interest in recent research on WDM networks has been to identify effective routing and wavelength assignment strategies for endto-end connections. Networks that comprise exclusively WC switches have immediate analogues in classical circuit switched networks, hence many issues about these networks are relatively well understood. Networks with limited wavelength converter or WS switches form a distinct class due to the wavelength continuity constraint, and appear to require more sophisticated analysis tools.

This paper focuses on dynamic wavelength assignment in WDM optical networks, and gives an overview of some recent results. The aim of the paper is to give a perspective on the subject and to illustrate a new method of analysis. Thus the articles cited here do not constitute an exhaustive list of references on wavelength assignment, however throughout the paper the reader is frequently referred to relevant survey papers for this purpose. It is assumed here that each end-to-end connection occupies a full wavelength, 
and that connections are routed over predetermined paths. The efficiency of an assignment strategy is considered in two categories depending on the performance metric and a corresponding modeling approach. The first approach assumes a probabilistic structure for the connection set-up/tear-down process: set-up requests arrive according to Poisson processes, and connections are torn-down after exponentially distributed holding times. The model therefore inherently involves blocking of requests due to capacity constraints, and the aim in wavelength assignment is to minimize the blocking probability. While the Poisson request arrival model seems possibly suitable for the optical networks of the future, contemporary optical networks serve aggregated traffic with inherently different statistics. In addition, blocking is highly undesirable in these networks due to the very high data rates carried on a single wavelength. In view of these the second approach adopts a deterministic analysis and seeks the minimum number of wavelengths required assure no blocking for a given class of dynamic scenarios.

The outline of the paper is as follows. Section 2 concerns probability of blocking. In Section 2.1 networks with WC switches are considered, and some results from classical circuit switching theory are cited. Wavelength assignment is not an issue here, however the cited results have implications in networks with WS switches. These networks are the subject of Section 2.2. A brief overview of simple assignment heuristics proposed in the literature is given, and some recent work on an asymptotically exact model is discussed. Section 3 summarizes some recent results on nonblocking WDM optical networks. We conclude by identifying some open issues in Section 4 .

\section{Loss Networks}

We consider a network with $J$ edges, each edge representing a fiber with $W$ wavelengths. The network serves a number of connection types, each of which is identified by the set of edges in its route. Connection requests of different types arrive according to statistically independent Poisson processes, with rate $\nu_{r}$ for connection type $r$. If the network has sufficient free capacity to accommodate a new connection request, the request is granted and the connection holds one wavelength on each fiber on its route for the duration of its holding time. It is assumed that wavelength reassignment is not allowed: each connection keeps the same wavelength assignment as it remains in the network. Holding times are independent and have exponentially distribution with mean 1 . Requests that cannot be granted are 
blocked and lost.

\subsection{Networks without wavelength continuity constraint}

If all network nodes are WC switches and the wavelength continuity constraint is not in effect, then a connection request is accepted if each link on its route has a free wavelength. Blocking in such networks has been studied extensively in the context of circuit switched telephone networks, and this section cites some results thereof. Let $R$ denote the collection of connection types, and the routes be represented by the incidence matrix $A=\left[A_{j, r}\right]_{J \times R}$, where $A_{j, r}=1$ if link $j$ belongs to route of type $r$, and $A_{j, r}=0$ otherwise. Hence $r=\left\{j \in J: A_{j, r}=0\right\}$. Denote by $n_{t}(r)$ the number of type $r$ connections in the network at time $t$, and define the vector $n_{t}=\left(n_{t}(r): r \in R\right)$. Let $\underline{W}$ denote a $|J|$ dimensional vector whose entries are $W$. The process $\left(n_{t}: t \geq 0\right)$ is Markovian with state space $S=\left\{n \in Z_{+}^{R}: A n \leq \underline{W}\right\}$. The equilibrium distribution of this process, $\theta$, is given by[3]

$$
\theta(n)=G^{-1} \prod_{r \in R} \frac{\nu_{r}^{n(r)}}{n(r) !} \quad n \in S
$$

Here $G$ is a normalizing constant which makes $\theta$ a probability vector.

The equilibrium distribution $\theta$ leads immediately to the blocking probability for type $r$ connections as

$$
B_{r}=\sum_{n: A n>\underline{W}-A e_{r}} \theta(n)
$$

where $e_{r}$ denotes a binary vector whose entries are zero except the $r$ th entry. However the task of evaluating this expression is \#P complete[4]; thus one usually has to seek accurate methods to approximate blocking probabilities.

An appealing independence assumption gives rise to a well-known reduced load approximation: Consider the hypothetical situation in which links blocked requests in a mutually independent manner. Let $L_{j}$ denote the probability that link $j$ has no free wavelengths. The rate of connection requests of each type at a link $i$ would then be thinned by a fraction $1-L_{j}$ at every other link $j$ on the route of that type. In particular, the process of connection requests seen by link $i$ would be a Poisson process with rate

$$
\rho_{i}=\sum_{r: i \in r} \nu_{r} \prod_{j \in r-\{i\}}\left(1-L_{j}\right)
$$


In turn, equation (1), applied to a single link, would imply that

$$
L_{i}=\left(\sum_{n=0}^{W} \frac{\rho_{i}^{n}}{n !}\right)^{-1} \frac{\rho_{i}^{W}}{W !} .
$$

The reduced load approximation is obtained by using

$$
E_{r}=1-\prod_{i \in r}\left(1-L_{i}\right)
$$

to approximate the blocking probability $B_{r}$. Equations (2)-(3) admit a unique solution and the approximation is well defined.

The reduced load approximation outlined above is surprisingly effective for networks employing fixed routing. The approximation gives asymptotically exact results in a limiting regime in which the number of wavelengths $W$ and the request arrival rates $\nu_{r}$ increase in proportion[3]. Variants of the method have been shown to perform well in the finite case also[5]. The reader is referred to [3] for an excellent discussion of reduced load approximations and further insight on blocking in classical loss networks.

\subsection{Networks with wavelength continuity constraint}

Quantifying the increase in blocking due to the wavelength continuity constraint has been a common theme in much of recent research. Several facts make this a challenging task. Determining the exact capacity of networks with WS switches appears difficult: whether a fixed set of connections can be established simultaneously in the network is a NP-complete problem [6]. For the dynamic case, a closed form expression for the steady state distribution can seldom be obtained for nontrivial topologies or reasonable wavelength assignment policies.

Addressing the fundamental limitations due to wavelength continuity entails having deeper insight on wavelength assignment. In a network of WS switches a wavelength may be shared by two connections only if the connections do not use the same fiber. Thus the wavelengths are reused spatially. A number heuristic wavelength assignment strategies proposed in the literature have been inspired by the informal observation that maximizing wavelength reuse lead to high utilization of network resources. Two such strategies are First-Fit [6], which selects the available wavelength with the smallest index, and Most-Used [7], which selects an available wavelength that is most utilized within the entire network. Both strategies implicitly 
promote packing connections into a small number of wavelengths so as to reserve free capacity for potential new requests.

The Max-Sum [8] strategy, which has a slightly different flavor than the previous two, selects a wavelength so as to reduce an explicit measure of blocking potential in the resulting state of the network. Simulations indicate that Max-Sum performs better than First-Fit and Most-Used strategies [8]. This is not totally unexpected since Max-Sum mimics a myopic optimization with regard to the future. In principle progressively better strategies can be obtained by extending the optimization horizon further. This entails formulating stochastic dynamic programs, however the dimension of the state space invalidates this approach for typical problems. One such formulation is adopted in [9] where policy iteration[10] is employed to improve the blocking performance of any given strategy. Here the cost-to-go functions of the original strategy are evaluated via simulations.

Majority of the analytic approaches employed in performance evaluation of networks of WS switches involve the assumption of link independence. This argument assumes that links have statistically independent states, and wavelengths on the same fiber have statistically independent occupancies. Clearly this is not the case under the general model of the present section. Typical applications of the link independence assumption do not entail a fixed point argument (see the list of references in [11]), hence these are fundamentally not analogous to the reduced load approximations of the previous section.

In principle a generalized form of the reduced load approximations may be incorporated in the analysis of the networks considered in this section by treating each fiber as $W$ parallel links with unit capacity. The flexibility of choosing among possible wavelengths in the original network then corresponds to alternate routing in the classical circuit switched counterpart. The asymptotic exactness of the reduced load approximation does not extend to networks with alternate routing[13], instead one can employ the generalized reduced load approximation that is formulated in [14]. This technique does not offer hard guarantees on accuracy, and the involved computational complexity tends to be high for large networks[15]. See [15], [16] and [17] for applications of this technique.

While the link independence assumption permits an explicit analysis and captures some salient features of routing and wavelength assignment, it fails to answer somewhat deeper questions due to the inherent oversimplification. [18] addresses this shortcoming by considering a different approximate model that accounts for links correlations. Analysis of the model reveals that the 
blocking performance depends on the ratio of the average route length to the average number of links shared by two intersecting routes. This result confirms earlier observations that the wavelength continuity constraint has a smaller impact in ring topologies compared to mesh topologies. For detailed discussions of heuristic algorithms and approximate models, the reader is referred to the survey articles $[11,19]$.

Significant insight can be gained on the network behavior by using approximate dynamic models that are exact in some asymptotic sense. The rest of this section concerns one such model due to [20] that provides information about the dynamic as well as the steady state behavior of the network. Consider a network whose topology is given by Figure 2. Let $W, \nu_{1}, \nu_{2}, \nu_{3}$ be positive numbers and let $\gamma$ be a scaling factor so that each fiber has $\lfloor\gamma W\rfloor$ wavelengths and type $r$ connections arrive at rate $\gamma \nu_{r}$. Each type 1 (respectively type 2) connection require one wavelength from fiber 1 (respectively fiber 2). Each type 3 connection requires one wavelength from each fiber, furthermore these wavelengths should be identical. The MostUsed wavelength assignment strategy is adopted: a type 1 (respectively type 2) connection is assigned an available wavelength that is already occupied on fiber 2 (respectively fiber 1), if such a wavelength exists at the time of arrival. If all available wavelengths are idle in both fibers, then one such wavelength is assigned arbitrarily.

For each time $t$ and type $r=1,2,3$ define

$$
\begin{aligned}
& X_{t}(r)=\text { number of type } r \text { connections in the network at time } t \\
& X_{t}(4)=\text { number of wavelengths available on both fibers at time } t
\end{aligned}
$$

and let $X_{t}=\left(X_{t}(1), X_{t}(2), X_{t}(3), X_{t}(4)\right)$. See Figure 3 for an illustration of these variables. Under Most-Used wavelength assignment the process $\left(X_{t}: t \geq 0\right)$ is Markovian. Its normalized version $\left(X_{t} / \gamma: t \geq 0\right)$, where division by $\gamma$ is understood to be componentwise, converges in probability as $\gamma$ grows towards infinity. The limit, denoted by $\left(x_{t}: t \geq 0\right)$, complies with the following differential equalities:

$$
\begin{aligned}
\dot{x}_{t}(1)= & \nu_{1} \pi_{x_{t}}(m(1)+m(3)>0)-x_{t}(1) \\
\dot{x}_{t}(2)= & \nu_{2} \pi_{x_{t}}(m(2)+m(3)>0)-x_{t}(2) \\
\dot{x}_{t}(3)= & \nu_{3} \pi_{x_{t}}(m(3)>0)-x_{t}(3) \\
\dot{x}_{t}(4)= & \left(2 W-x_{t}(1)-x_{t}(2)-x_{t}(3)-2 x_{t}(4)\right)-\nu_{3} \pi_{x_{t}}(m(3)>0) \\
& -\nu_{1} \pi_{x_{t}}(m(1)=0, m(3)>0)-\nu_{2} \pi_{x_{t}}(m(2)=0, m(3)>0)
\end{aligned}
$$




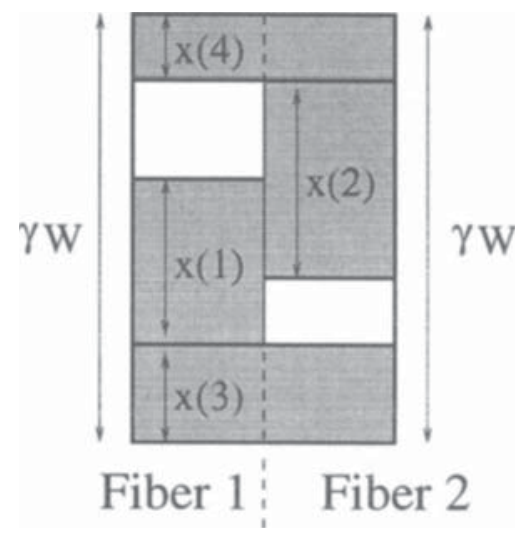

Figure 3: A typical state of the network of Figure 2.

Here $\pi_{x_{t}}$ denotes an equilibrium distribution of a Markov process $(m(1), m(2)$ $m(3))$ that mimics the free wavelength process in the network. Informally, $m(1)$ (respectively $m(2)$ ) represents the number of wavelengths that are available only on fiber 1 (respectively fiber 2), and $m(3)$ represents the number wavelengths that are available on both fibers. Note that the actual free wavelength process is not Markovian: its instantaneous transition rates at time $t$ depend on the value $X_{t}$. These rates are thus proportional to $\gamma$, and in the limit of large $\gamma$ the free wavelength process settles to equilibrium before the value of the normalized process $X_{t} / \gamma$ changes. The resulting equilibrium distribution is captured by the process $(m(1), m(2), m(3))$ whose transition rates are given by Table 1 . Here and in the rest of the paper $I$ denotes the indicator function, which is 1 if the condition in the argument holds, and 0 otherwise. Since the total number of wavelengths is unbounded in the limit, the coordinates of the process $(m(1), m(2), m(3))$ may take on the value $\infty$. Thus this process is inherently reducible and possesses multiple equilibrium distributions. Solving for the trajectories (4)-(7) entails identifying the right distribution at every $t$, which is a nontrivial procedure.

Equations (4)-(7) can be interpreted as follows. Equation (4) indicates that type 1 connections are admitted to the network at a rate proportional to the probability that there is a free wavelength on fiber 1 . The total rate of termination of type 1 connections is proportional to the total number of such connections, since each connection terminates independently of the others. Equations (5) and (6) are interpreted similarly for type 2 and type 3 connections respectively. The number of wavelengths that are free on both 


\begin{tabular}{r|l} 
Jump & Rate \\
\hline$(1,0,0)$ & $x_{t}(1)+x_{t}(2)+x_{t}(3)+x_{t}(4)-W$ \\
$(0,1,0)$ & $x_{t}(1)+x_{t}(2)+x_{t}(3)+x_{t}(4)-W$ \\
$(0,0,1)$ & $2 W-\left(x_{t}(1)+x_{t}(2)+x_{t}(3)+2 x_{t}(4)\right)$ \\
$(-1,0,0)$ & $\nu_{1} I\{m(1)>0\}$ \\
$(0,1,-1)$ & $\nu_{1} I\{m(1)=0, m(3)>0\}$ \\
$(0,-1,0)$ & $\nu_{2} I\{m(2)>0\}$ \\
$(1,0,-1)$ & $\nu_{2} I\{m(2)=0, m(3)>0\}$ \\
$(0,0,-1)$ & $\nu_{3} I\{m(3)>0\}$
\end{tabular}

Table 1: Transition rates of the process $(m(1), m(2), m(3))$.

fibers increase either when a type 3 connection terminates, or when a type 1 (respectively type 2) connection terminates while its assigned wavelength is free on fiber 2 (respectively fiber 1 ). This is captured by the first term in equation (7). This number decreases upon a type 3 connection arrival or upon a type 1 or type 2 arrival while all available wavelengths are those that are free on both fibers, as indicated by the remaining terms in equation (7).

We next examine the solutions of the limiting model (4)-(7). Figures 4.a4.d depict the trajectories $\left(x_{t}: t \geq 0\right)$ starting from an empty system, for four different sets of parameters. We concentrate on the steady state system behavior under the following classification:

1) Underloaded network $\left(\nu_{1}+\nu_{3} \leq W, \nu_{2}+\nu_{3} \leq W\right)$ : In the underloaded case the capacity constraint due to finite $W$ is not binding in the considered limit. Thus connections of each type $r=1,2,3$ are virtually undisturbed by the existence other types, and their normalized number converge exponentially to $\nu_{r}$ in steady state. Furthermore the normalized number of wavelengths that are available on both fibers converge to $\min _{r \in\{1,2\}}\left(W-\nu_{r}-\nu_{3}\right)$, the largest possible value. Hence in the particular topology considered here, Most-Used assignment suffices to pack the utilized wavelengths as closely as possible.

2) Partially overloaded network $\left(\nu_{1}+\nu_{3}>W, \nu_{2}+\nu_{3} \leq W\right)$ : In the underloaded fiber, in this case fiber 2 , there is virtually no contention for capacity and therefore there is no blocking. In turn the normalized number of type 2 connections evolve as in the underloaded case considered above, converging to $\nu_{2}$ in equilibrium. Type 3 connections however are subject to blocking since they share the overloaded fiber 1 with type 1 connections. 


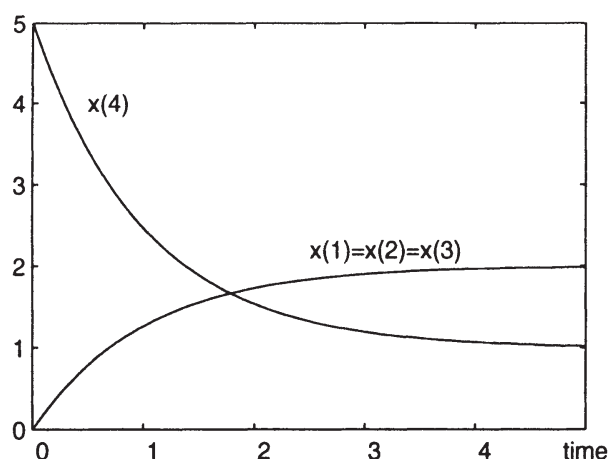

(a)

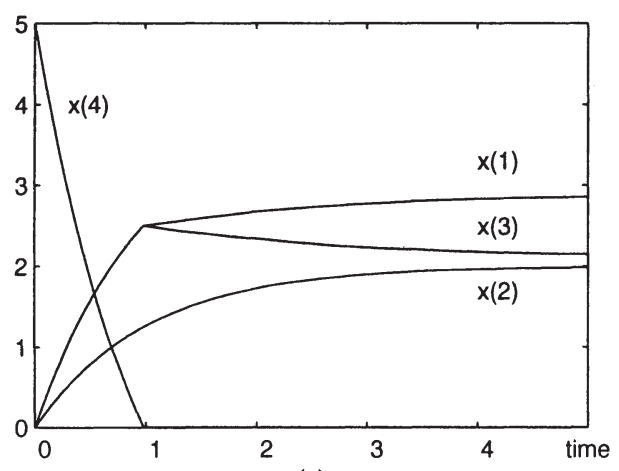

(c)

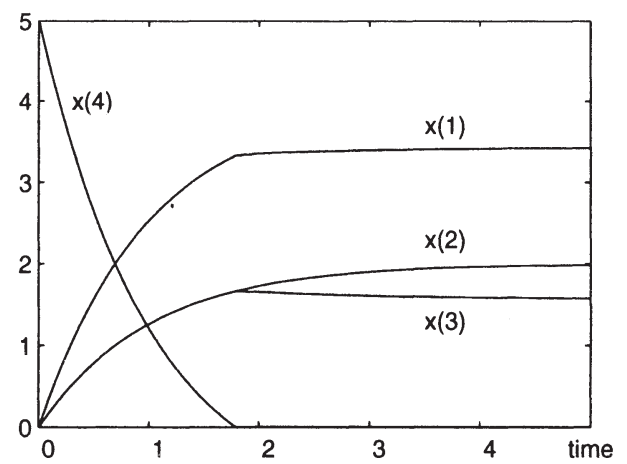

(b)

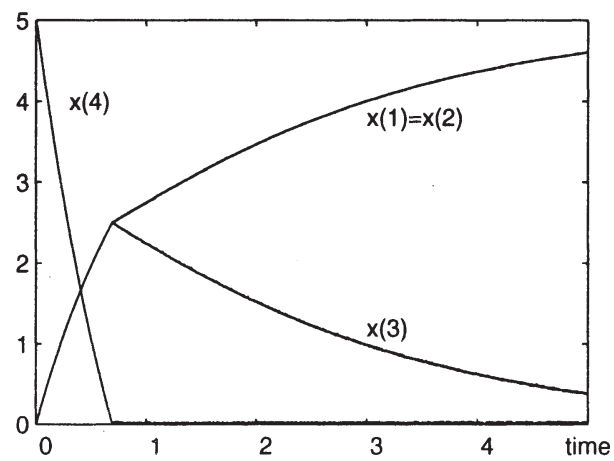

(d)

Figure 4: Limiting trajectories for four sets of system parameters that lead to qualitatively different behavior: (a) $\nu_{1}=2, \nu_{2}=2, \nu_{3}=2, W=5$, (b) $\nu_{1}=4, \nu_{2}=2, \nu_{3}=2, W=5$, (c) $\nu_{1}=4, \nu_{2}=2, \nu_{3}=4, W=5$, (d) $\nu_{1}=5, \nu_{2}=5, \nu_{3}=5, W=5$. All considered cases start with empty system.

On the one hand, type 3 connections have a disadvantage against type 1 connections, since they may not use the wavelengths occupied by type 2 connections on fiber 2 . On the other hand, these wavelengths are selected with priority by type 1 connections, due to the Most-Used assignment rule.

The trajectory $\left(x_{t}: t \geq 0\right)$ converges to the limit point

$$
x_{\infty}=\left(\nu_{1} \pi(m(1)+m(3)>0), \nu_{2}, \nu_{3} \pi(m(3)>0), 0\right)
$$

where $\pi$ is the equilibrium distribution of the ergodic Markov process with values in two-dimensional nonnegative integers, and transition rates as given by Table 2. A fairly accurate approximation to $x_{\infty}$ is obtained via an in- 


\begin{tabular}{r|l} 
Jump & Rate \\
\hline$(1,0)$ & $\nu_{2}$ \\
$(-1,0)$ & $\nu_{1} I\{m(1)>0\}$ \\
$(0,1)$ & $W-\nu_{2}$ \\
$(0,-1)$ & $\nu_{3} I\{m(3)>0\}+\nu_{1} I\{m(1)=0, m(3)>0\}$
\end{tabular}

Table 2: Transition rates of the Markov process $(m(1), m(3))$ that determines the limit point of the partially overloaded system under Most-Used assignment.

\begin{tabular}{r|l} 
Jump & Rate \\
\hline$(1,0)$ & $\nu_{2}$ \\
$(-1,0)$ & $\nu_{1} I\{\hat{m}(1)>0\}$ \\
$(0,1)$ & $W-\nu_{2}$ \\
$(0,-1)$ & $\left(\nu_{3}+\nu_{1} \pi(m(1)=0)\right) I\{\hat{m}(3)>0\}$
\end{tabular}

Table 3: Transition rates of the Markov process $(\hat{m}(1), \hat{m}(3))$ that is used in obtaining the approximate limit point (8). Note that $\pi(m(1)=0)=$ $1-\left(\nu_{2} / \nu_{1}\right)$ by Table 2 .

dependence assumption that is analogous to those used in the analysis of loss networks with state dependent routing[15]. The approximation entails replacing $\pi$ with the equilibrium distribution of the Markov process that has transition rates given by Table 3 , and results in the approximate limit point

$$
\left(\nu_{2}+\left(W-\nu_{2}\right) \frac{\nu_{1}-\nu_{2}}{\nu_{3}+\nu_{1}-\nu_{2}}, \nu_{2},\left(W-\nu_{2}\right) \frac{\nu_{3}}{\nu_{3}+\nu_{1}-\nu_{2}}, 0\right) .
$$

This point reflects an operating regime in which a fraction $\nu_{2} / \nu_{1}$ of all type 1 arrivals are assigned wavelengths that are occupied by type 2 connections, and the remaining fraction $\left(W-\nu_{2}\right) / W$ of all wavelengths on fiber 1 is shared between the type 3 and excess type 1 connections, in proportion with the respective arrival rates $\nu_{3}$ and $\nu_{1}-\nu_{2}$. Though intuitive, the approximation (8) is not exact in that it slightly underestimates the fair share of type 3 connections.

3) Totally overloaded network $\left(\nu_{1}+\nu_{3}>W, \nu_{2}+\nu_{3}>W\right)$ : Let us start with the case when $\nu_{2}<\nu_{1} \leq W$. There is competition for free wavelengths 
on both fibers, however type 3 connections face a harsher contention on fiber 1. In turn enough of type 3 arrivals are knocked out by type 1 connections so that type 2 connections virtually enjoy the perception of an underloaded fiber. Therefore in steady state the normalized number of type 2 connections in the network converge to $\nu_{2}$. The argument presented for the partially overloaded case above now applies, and it implies that the steady state normalized numbers are again given by the vector $x_{\infty}$ above. This discussion holds as $\nu_{2}$ increases towards $\nu_{1}$, in particular if $\nu_{1}=\nu_{2} \leq W$ the limit point is $x_{\infty}=\left(\nu_{1}, \nu_{1}, W-\nu_{1}, 0\right)$. Note that as $\nu_{1}, \nu_{2}$ increase towards $W$, the number of type 3 connections carried by the network vanishes. In the case when $\min \left\{\nu_{1}, \nu_{2}\right\} \geq W$ the network operates around the normalized state $(W, W, 0,0)$, and type 3 connections are virtually locked out of the network. This is an extreme manifestation of the fairness issue that has been raised in several works[11].

The asymptotic exactness of the model (4)-(7) is essentially a functional law of large numbers, hence the error in the approximation is $o(\gamma)$. For the unnormalized system, the model should be regarded as a qualitative description of the expected system dynamics. In the particular context considered here, this description yields insight on the transient and static behavior of the network, which is not captured via link independence or reduced load approximations.

From a practical point of view, perhaps the most significant of the above results is that the Most-Used strategy has near optimal performance in utilizing the available wavelengths in the considered topology. In particular, in the considered limit of large capacities and load, the Most-Used strategy has the best possible underload condition in which there is virtually no blocking in the network. It is interesting to see if this property extends to more general network topologies.

\section{Nonblocking Networks}

In this section we give a brief summary of results on requirements for no blocking. Earlier work on these networks concerned determining the minimum number of wavelengths to satisfy static demand patterns. A popular static pattern is a permutation, in which each node is the origin and the destination of exactly one connection. Bounds on the necessary number of wavelengths required to establish a permutation routing for general topologies are provided in $[21,22]$. Similar results are given in [23] for bounded 
degree topologies, and a randomized algorithm is given to route the connections with close to minimum number of wavelengths with high probability.

The number of wavelengths required to satisfy dynamic traffic patterns has been recently studied for ring topologies[24]. Namely a ring network with $N$ WS nodes is considered. The traffic on the ring comprises end-toend connections whose routes are predetermined in advance. It is shown that if the routes and the connection set-up/tear-down times are arbitrary subject to the condition that at any time no fiber carries more than $L$ active connections, then a reservation based assignment strategy guarantees no blocking with roughly $L+L \log _{2} N$ wavelengths. With regard to a lower bound, there exists an explicit set-up/tear-down sequence for which any wavelength assignment algorithm would need at least $0.5 L \log _{2} N$ wavelengths for no blocking. The First-Fit strategy is shown to guarantee noblocking with $2.52 L \log _{2} N+5 L$ wavelengths. For the case of limited wavelength conversion, [24] provides an explicit construction of a conversion scheme in which each wavelength can be converted to one of two specified wavelengths. With this construction the number of required wavelengths reduce to $L \log _{2} \log _{2} L+4 L$.

Results of [24] indicate that satisfying dynamic connection patterns on the ring via WS switches proves to be very demanding on network resources: Clearly $L$ wavelengths suffice to guarantee no blocking with WC switches. Static patterns do not require more than $2 L-1$ wavelengths even with WS switches[24]. However dynamic patterns under the wavelength continuity constraint result in significant underutilization of wavelengths as summarized above. On the positive side, even a small degree of limited wavelength conversion helps reduce the wavelength requirement considerably, in agreement with results reported elsewhere[11, 19].

\section{Conclusion}

This paper concerned some current issues in the analysis and synthesis of dynamic wavelength assignment policies for optical WDM networks. We conclude by providing a brief list of open issues. Accurate analysis methodologies are needed to shed more light on the network behavior under the wavelength continuity constraint, and possibly to lead to identifying effective assignment strategies. Limited wavelength conversion appears to be a promising solution to increase wavelength utilization and deserves further investigation. Effective wavelength conversion and assignment methods, as 
well as fundamental limits on the gains obtained by limited conversion are unknown for general topologies. Finally, in current WDM systems link capacities are increased by using additional fibers. The crosspoint complexity per wavelength of a WS switch increases with the number of incident fibers, indicating possible improvement in wavelength utilization. Numerical studies[19] support this observation, and warrant further investigation of networks with multiple fibers per link.

\section{References}

[1] P. E. Green, Fiber optic networks, (Prentice-Hall, 1993).

[2] J. M. H. Elmir, and H. T. Muftah, All-optical wavelength conversion: technologies and applications in DWDM networks, IEEE Communication Magazine Vol. 38 No. 3 (2000) pp. 86-92.

[3] F. P. Kelly, Loss networks, Annals of Applied Probability, Vol. 1 (1991) pp. 319-378.

[4] G. Louth, M. Mitzenmacher, and F. P. Kelly, Computational complexity of loss networks, Theoretical Computer Science, Vol. 125 (1994) pp. 4559.

[5] S. Chung and K. W. Ross, Reduced load approximations for multirate loss networks, IEEE Transactions on Communications, Vol. 41, No. 8 (1993) pp. 1222-1231.

[6] I. Chlamtac, A. Ganz, and G. Karmi, Lightpath communications: an approach to high bandwidth optical WANs, IEEE Transactions on Communications, Vol. 40 No. 7 (1992) pp.1171-1182.

[7] A. Mokhtar and M. Azizoglu, Adaptive wavelength routing in all-optical networks, IEEE/ACM Transactions on Networking, Vol. 6. No. 2 (1998) pp. 197-206.

[8] S. Subramaniam and R. A. Barry, Wavelength assignment in fixed routing WDM networks, Proceedings of IEEE ICC (1997) pp. 406-410.

[9] E. Hyytia and J. Virtamo, Dynamic routing and wavelength assignment using first policy iteration, Fifth Symposium on Computers and Communications ISCC'00 (2000). 
[10] S. M. Ross, Introduction to stochastic dynamic programming, (Academic Press, 1983).

[11] E. Karasan and E. Ayanoglu, Performance of WDM transport networks, IEEE Journal of Selected Areas in Communications, Vol.16 No. 7 (1998) pp. 1081-1096.

[12] E. Karasan and E. Ayanoglu, Effects of wavelength routing and selection algorithms on wavelength conversion gain in WDM optical networks, IEEE/ACM Transactions on Networking, Vol. 6 No. 2 (1998) pp. 186196.

[13] P. J. Hunt and T. G. Kurtz, Large loss networks, Stochastic Processes and their Applications, Vol. 53 (1994) pp. 363-378.

[14] F. P. Kelly, Routing and Capacity Allocation in Networks with Trunk Reservation, Mathematics of Operations Research, Vol. 15 (1990) pp. 771-792.

[15] S. P. Chung, A. Kashper, and K. W. Ross, Computing Approximate Blocking Probabilities for Large Loss Networks with State-Dependent Routing, IEE/ACM Transactions on Networking, Vol. 1 (1993) pp. 105115.

[16] D. Mitra, R. J. Gibbens, and B. D. Huang, State-Dependent Routing on Symmetric Loss Networks with Trunk Reservations - I, IEEE Transactions on Communications, Vol. 41 (1993) pp. 400-411.

[17] D. Mitra, and R. J. Gibbens, State-Dependent Routing on Symmetric Loss Networks with Trunk Reservations, II: Asymptotics, Optimal Design, Ann. Oper. Res., Vol. 35 (1992) pp. 3-30.

[18] R. A. Barry and P. A. Humblet, Models of blocking probability in alloptical networks with and without wavelength changers, IEEE Journal of Selected Areas in Communications, Vol. 14 No. 5 (1996) pp. 858-867.

[19] J. M. Yates, M. P. Rumsewitz, and J. P. R. Lacey, Wavelength converters in dynamically reconfigurable WDM networks, IEEE Communications Surveys, http://www.comsoc.org/pubs/surveys, (1999).

[20] M. Alanyali, Asymptotically exact models for some wavelength assignment strategies in a two-hop optical network, (Technical Report, Electrical and Electronics Eng. Dept., Bilkent University), (2000). 
[21] A. Aggarwal, A. Bar-Noy, D. Coppersmith, R. Ramaswami, B. Schieber, and M. Sudan, Efficient routing and scheduling algorithms for optical networks, Proceedings of ACM-SIAM SODA, (1994) pp. 412-423.

[22] R. A. Barry and P. A. Humblet, On the number of wavelengths and switches in all-optical networks, IEEE Transactions on Communications, Vol. 42 No. 2/3/4 (1994) pp. 583-591.

[23] P. Raghavan and E. Upfal, Efficient routing in all-optical networks, Proceedings of 26th ACM Symp. Theory of Computing, (1994) pp. 134143.

[24] O. Gertsel, G. Sasaki, S. Kutten, and R. Ramaswami, Worst-case analysis of dynamic wavelength allocation in optical networks, IEEE/ACM Transactions on Networking, Vol. 7 No. 6 (1999) pp.833-845. 